\title{
The quantum vacuum at the foundations of classical electrodynamics
}

\author{
G. Leuchs • A.S. Villar • L.L. Sánchez-Soto
}

Received: 30 April 2010 / Published online: 29 May 2010

(C) The Author(s) 2010. This article is published with open access at Springerlink.com

\begin{abstract}
In the classical theory of electromagnetism, the permittivity $\varepsilon_{0}$ and the permeability $\mu_{0}$ of free space are constants whose magnitudes do not seem to possess any deeper physical meaning. By replacing the free space of classical physics with the quantum notion of the vacuum, we speculate that the values of the aforementioned constants could arise from the polarization and magnetization of virtual pairs in vacuum. A classical dispersion model with parameters determined by quantum and particle physics is employed to estimate their values. We find the correct orders of magnitude. Additionally, our simple assumptions yield an independent estimate for the number of charged elementary particles based on the known values of $\varepsilon_{0}$ and $\mu_{0}$ and for the volume of a virtual pair. Such an interpretation would provide an intriguing connection between the celebrated theory of classical electromagnetism and the quantum theory in the weak-field limit.
\end{abstract}

In classical electrodynamics, a dielectric medium becomes polarized in the presence of an electric field. A weak electric field slightly displaces the electronic clouds from their

G. Leuchs $(\varangle) \cdot$ A.S. Villar · L.L. Sánchez-Soto

Max Planck Institut für die Physik des Lichts,

Günther-Scharowsky-Str. 1/Bau 24, 91058 Erlangen, Germany

e-mail: gerd.leuchs@mpl.mpg.de

G. Leuchs · A.S. Villar

Institut für Optik, Information und Photonik, Universität Erlangen-Nürnberg, Staudtstr. 7/B2, 91058 Erlangen, Germany

L.L. Sánchez-Soto

Departamento de Óptica, Facultad de Física, Universidad

Complutense, 28040 Madrid, Spain binding nuclei, producing tiny atomic and molecular dipoles in the material. The macroscopic effect, averaged over a region large compared to the atomic dimensions, is the appearance of an induced electric field. The strength of this response depends on how susceptible to displacement the atomic dipoles are and on how much space they occupy.

Putting it in more quantitative terms, in an isotropic medium the polarization $\mathbf{P}$ is proportional to the external electric field $\mathbf{E}$ through the relation $\mathbf{P}=\chi \varepsilon_{0} \mathbf{E}$, where $\chi$ is the linear susceptibility of the material and $\varepsilon_{0}$ is the permittivity of free space. The constant $\varepsilon_{0}$ has the unimpressive role of matching units, just 'happening to have the value' $\varepsilon_{0}=8.8542 \times 10^{-12} \mathrm{~A} \mathrm{~s} /(\mathrm{V} \mathrm{m})$ [1]. The expression for the magnitude of $\mathbf{P}$, which connects the microscopic dipoles to their macroscopic effect, reads

$P=\frac{\wp}{V}$

where $\wp$ is the dipole moment and $V$ is the effective volume per dipole.

It is customary to define the electric displacement as

$\mathbf{D}=\varepsilon_{0} \mathbf{E}+\mathbf{P}$,

since it turns out to be independent of the induced charge [2]. In the particular case of an isotropic linear dielectric, (2) yields $\mathbf{D}=\varepsilon \varepsilon_{0} \mathbf{E}$, where $\varepsilon=(1+\chi)$ is the relative permittivity of the medium.

The electric displacement D enjoys no better status among us physicists than that of $\varepsilon_{0}$. It is mostly seen as a mathematical tool of limited use, because it would combine two physically different quantities [2]. In fact, the electric field is the force per unit charge, whilst the spatial variation of the polarization is the induced charge. Sometimes, 
the electric displacement is even considered completely dispensable [3], since the condition $\boldsymbol{\nabla} \times \mathbf{D}=0$ does not apply in general-in contrast to the case of $\mathbf{E}$, a scalar potential cannot be associated with $\mathbf{D}$.

In this paper, we offer a physical interpretation of $\varepsilon_{0}$ and thus of $\mathbf{D}$ [4-6]. We discuss how (2) might hint at fundamental physical aspects of the electromagnetic field itself if, in the light of its second term, we interpret the first term as also originating from a polarized medium, so that $\mathbf{D}$ is the total polarization. That would put $\varepsilon_{0} \mathbf{E}$ and $\mathbf{P}$ on an equal footing. ${ }^{1}$ That interpretation is preposterous in classical electromagnetism, since the vacuum is defined as the emptiness. In quantum theory, however, that is not the case, and virtual particle pairs making up the vacuum are expected to show a response to an applied field. In analogy to $\varepsilon \varepsilon_{0}$ for a material medium, $\varepsilon_{0}$ itself could be associated with the polarization of the quantum vacuum $P_{0}=\varepsilon_{0} E$, a concept deeply rooted in quantum electrodynamics [7, 8]. We later extend the discussion to magnetic phenomena, linking the permeability of free space $\mu_{0}$ to the magnetization of virtual pairs in vacuum.

The presence of an external electromagnetic field disturbs the stability of the vacuum as the ground state of all fields. In this case, virtual particles must be considered to account for the possible physical processes in vacuum. Phenomena such as pair creation, vacuum birefringence, and light-light scattering are well established for strong fields [9-12], with amplitudes larger than $E_{S}=m^{2} c^{3} / e \hbar \approx 10^{18} \mathrm{~V} / \mathrm{m}$. This is exactly the expression for the critical field first pointed out by Niels Bohr, as acknowledged by F. Sauter. ${ }^{2}$ In the literature, $E_{S}$ is often referred to as the Schwinger field [12]. These effects illustrate the nonlinear properties of the vacuum $[7,8]$.

Here we assume the limit of weak fields and focus on the linear response. The question we pose is: could the vacuum quantum fluctuations be subtly embedded in the classical theory? If yes, one piece of evidence could be the existence of physical constants whose numerical values are simply determined experimentally, but which would emerge naturally from the quantum theory. We speculate whether the permittivity and the permeability of free space could be such quantities. In the remainder of this paper, we will estimate the values of $\varepsilon_{0}$ and $\mu_{0}$ based on simple semi-classical assumptions.

We start by considering the electrical properties of the quantum vacuum. An external electric field interacting with

\footnotetext{
${ }^{1}$ In Gaussian units, (2) reads $\mathbf{D}=\mathbf{E}+4 \pi \mathbf{P}$, so that $\varepsilon_{0}$ is replaced by the constant ' 1 .' In the language of Gaussian units, this paper discusses the physical significance of this ' 1 .'

${ }^{2}$ See second footnote on p. 743 of [10]: "I would like to thank Prof. Heisenberg for kindly informing me about this hypothesis of N. Bohr."
}

the virtual electron-positron pairs polarizes them. The magnitude of the dipole moment $\wp=e x$ induced on one virtual pair (where $e$ is the electron charge and $x$ is the displacement) can be computed by considering the virtual pair as a harmonic oscillator, such that in the quasi-static limit

$m \omega_{0}^{2} x=e E$

where $m$ is the electron mass and $\omega_{0}$ is the natural resonance frequency. As customary in this sort of semiclassical treatment, valid when the excitation of the quantum oscillator is very small (or equivalently $E \ll E_{S}$ ), the resonance frequency is determined by the energy associated with the quantum transition. The two-level system under consideration is formed by the ground state of the virtual pairs and the real positronium atom as the excited state [13]. The energy gap is denoted $\mathcal{E}_{\text {gap }}$, so that $\omega_{0}=\mathcal{E}_{\text {gap }} / \hbar$, and it is expected to be of the order of the positronium rest energy. In addition, the quasistatic regime requires that the frequency $\omega$ of the classical field $E$ be much smaller than the oscillator resonance frequency $\omega_{0}$. That means we are considering a low-energy field probing pair creation as a non-resonant effect (classical field). Inserting this relation for $\omega_{0}$ into (3) to determine the displacement, one obtains the induced dipole moment

$\wp=\frac{e^{2}}{m \omega_{0}^{2}} E$.

The magnitude of the vacuum polarization given by (1) depends on the effective volume per dipole $V=r^{3}$. In theoretical investigations [14], it is generally accepted that the appropriate length scale for a virtual electron-positron pair in vacuum is the Compton wavelength ${ }^{3} \lambda_{c}=\hbar / m c$. With these considerations, (1) results in the vacuum polarization $^{4}$

$P_{0}=\frac{e^{2}}{m \omega_{0}^{2} r^{3}} E$.

The induced polarization is proportional to the electric field, as expected. Assigning the quantity multiplying $E$ to the

\footnotetext{
${ }^{3}$ The uncertainty principle also offers an estimate for this quantity, by the condition $\Delta x \Delta p \geq \hbar / 2$, where $\Delta x$ and $\Delta p$ respectively denote the position and the momentum uncertainties. Taking the momentum $\Delta p=\hbar \omega_{0} / c$ necessary to close the rest energy gap would yield $\Delta x \geq$ $c / 2 \omega_{0}$, which is of the order of the Compton wavelength. The exact choice on how to relate $\Delta x$ to the dipole volume is arbitrary to a large extent when based on the uncertainty inequality.

${ }^{4}$ The same result would be derived in Gaussian units.
} 
vacuum polarizability, we arrive at ${ }^{5}$

$\tilde{\varepsilon}_{0}=\frac{e^{2}}{m \omega_{0}^{2} r^{3}}$.

For the permeability of free space $\mu_{0}$, we attempt a similar procedure to estimate its value by associating this quantity to the linear magnetic response of the quantum vacuum. Here we encounter the added difficulty that some aspects of the material magnetization arise from pure quantum effects without classical explanation. One must resort in this case to crude semi-classical assumptions that, nevertheless, have often proved useful in determining the correct order of magnitude of magnetic effects [15]. We will continue our analysis adapting results from quantum mechanics to obtain $\mu_{0}$ from a seemingly naive classical picture.

The magnetic counterpart of (2) is

$\mathbf{H}=\frac{1}{\mu_{0}} \mathbf{B}-\mathbf{M}$

where $\mathbf{M}$ is the magnetization and $\mathbf{H}$ is sometimes called the 'magnetic field,' although we will simply call it $\mathbf{H}$ and reserve the term for $\mathbf{B}$. In analogy to $\mathbf{D}$, the vector $\mathbf{H}$ isolates the contribution from the free current to the total magnetic field. The expression above hints at associating $\mu_{0}$ to the inverse of the vacuum magnetic response.

Analogously to the polarization, the magnitude of $\mathbf{M}$ can be calculated from the microscopic magnetic dipole moment $\mathcal{M}$ and its effective volume using

$M=\frac{\mathcal{M}}{V}$

where for consistency the same volume used for $\wp$ is assumed.

An external magnetic field applied to the vacuum induces an electric field vortex which accelerates the virtual electron and positron in opposite directions. This electric field $E_{i}$ induced in a circular orbit fulfills

$E_{i}=-\frac{r}{2} \dot{B}$,

\footnotetext{
${ }^{5}$ Substituting typical numbers in (6), for instance $\hbar \omega_{0}=2 m c^{2}$ as the rest energy of the positronium and $r=\lambda_{c} / 2$, one would get $\tilde{\varepsilon}_{0}=$ $2 e^{2} / \hbar c=1.62 \times 10^{-12} \mathrm{~A} \mathrm{~s} /(\mathrm{V} \mathrm{m})$. This number underestimates the correct value by one order of magnitude. However, apart from numerical prefactors which could possibly change by a small amount, we note that the expression for $\tilde{\varepsilon}_{0}$ does not depend on the rest mass if the energy gap is proportional to the latter. As discussed in what follows, this fact hints at having to sum the contributions from all elementary particles with electric charge, in this manner reinterpreting the above value of $\tilde{\varepsilon}_{0}$ as the partial contribution from the virtual electron-positron pairs. This approach provides an estimate for the number of elementary pairs contributing to the vacuum response.
}

where the dot denotes the time derivative. It generates a torque that increases the electron angular momentum $J$ by

$\Delta J=\frac{e r^{2}}{2} B$.

According to quantum mechanics, the magnetic moment and the angular momentum relate to each other through the expression

$M=\frac{g e}{2 m} J$,

where $g$ is the Landé factor. A completely orbital or diamagnetic contribution would render $g=1$, whilst $g=2$ would correspond to a pure spin or paramagnetic contribution. We choose the latter possibility for definiteness. Due to symmetry and simplicity, the angular momentum state of the virtual pair is assumed a singlet, in which case the positron contributes the same amount as the electron to the magnetic response. These particles have thus opposite spins and orbital angular momenta, but because of their opposite charges, the individual responses combine positively. Substituting the angular momentum variation given by (10) in (11), and multiplying by a factor 2 to account for the positron contribution, the induced dipole moment is

$\mathcal{M}=\frac{e^{2} r^{2}}{m} B$,

whence we get

$\tilde{\mu}_{0}=\frac{m r}{e^{2}}$.

The expressions for $\tilde{\varepsilon}_{0}$ and $\tilde{\mu}_{0}$ must be compatible with the Maxwell equations. Imposing $\tilde{\varepsilon}_{0} \tilde{\mu}_{0}=1 / c^{2}$, we find a relation between the transition energy $\mathcal{E}_{0}$ and the radius $r$. Moreover, the product $\tilde{\varepsilon}_{0} \tilde{\mu}_{0}$ does not depend on the volume of the virtual pair since it cancels out in the ratio,

$c^{2}=\frac{1}{\tilde{\varepsilon}_{0} \tilde{\mu}_{0}}=\frac{\mathcal{M} / B}{\wp / E}$,

implying that in our model the speed of light is a consequence of the magnetic and electric responses of each virtual pair locally. Using (4) and (12), the radius associated with the volume of the virtual pair then reads

$r=\frac{c}{\omega_{0}}$

Consistency with the Maxwell equations therefore results, by inserting (15) into (6), in the following expression for $\tilde{\varepsilon}_{0}\left(\right.$ and $\tilde{\mu}_{0}=1 / \tilde{\varepsilon}_{0} c^{2}$ ),

$\tilde{\varepsilon}_{0}=\frac{\hbar \omega_{0}}{m c^{2}} \frac{e^{2}}{\hbar c}$. 
This equation can be written in terms of the fine structure constant $\alpha=e^{2} /\left(4 \pi \varepsilon_{0} \hbar c\right)$ and the known value of $\varepsilon_{0}$ as ${ }^{6}$

$\tilde{\varepsilon}_{0}=4 \pi \alpha \frac{\hbar \omega_{0}}{m c^{2}} \varepsilon_{0}$.

This expression shows that our simple model supplies numerical values $\tilde{\varepsilon}_{0}$ and $\tilde{\mu}_{0}$ lying surprisingly close to the correct values ${ }^{7} \varepsilon_{0}$ and $\mu_{0}$, in this manner supporting their novel physical interpretation. Looking more closely, however, one notices that (16) tends to underestimate the correct $\varepsilon_{0}$ and overestimate $\mu_{0}$ for sensible choices of $\hbar \omega_{0}$. Also an astonishing property of $\tilde{\varepsilon}_{0}$ and $\tilde{\mu}_{0}$ becomes apparent: they are independent of the mass if, as expected, the energy gap depends linearly on $m$. Indeed, one might be tempted to assume that only the lightest elementary charged particles would contribute to the vacuum polarization, since their virtual excitation would demand the smallest amount of energy. But the independence of mass brings us to the intriguing conclusion that all charged elementary particle pairs should contribute more or less equally to the vacuum response. Equation (16) would then represent only one partial contribution to the vacuum polarization. The total response would be the sum of all partial contributions $[16,17]$, but weighted by the square of the charges $q_{j}$, where the index $j=1,2, \ldots$ stands for the different virtual elementary particle pairs. Equation (16) is modified accordingly to

$\tilde{\varepsilon}_{0}^{\text {total }}=4 \pi \alpha \frac{\hbar \omega_{0}}{m c^{2}} \sum_{j}\left(\frac{q_{j}}{e}\right)^{2} \varepsilon_{0}$.

We note in passing that the speed of light is independent of the number of particles, since the latter cancels out in (14). The equality between $\tilde{\varepsilon}_{0}$ and $\varepsilon_{0}$ imposes a relation between the energy gap and the number of elementary pairs, supplying an estimate for the latter,

$\sum_{j}\left(\frac{q_{j}}{e}\right)^{2}=\frac{1}{4 \pi \alpha} \frac{m c^{2}}{\hbar \omega_{0}}$

The choice $\hbar \omega_{0}=2 m c^{2}$ would suggest around ten contributing pairs with the electron charge.

In conclusion, our aim has been to demonstrate how a few reasonable assumptions suffice to derive the correct orders of magnitude of $\varepsilon_{0}$ and $\mu_{0}$, providing also a physical meaning to these quantities. ${ }^{8}$ Whilst regarded in classical

\footnotetext{
${ }^{6}$ In Gaussian units, the fine structure constant is $\alpha=e^{2} /(\hbar c)$, and the result does not depend on the unit system.

${ }^{7}$ Supposing $\hbar \omega_{0}=2 m c^{2}$, one obtains the deviation factor $4 \pi \alpha \hbar \omega_{0} / m c^{2} \approx 1 / 10$. We think this is surprisingly close considering the crude semi-classical model employed.

${ }^{8} \mathrm{~A}$ first step in a more quantitative model would take into account the distinction between $r^{2}$ in (12) and the average orbital radius needed for
}

electromagnetism as constants deprived of deeper physical meaning, in the light of the quantum theory they would be connected to fundamental physical processes, the polarization and the magnetization of virtual pairs in vacuum in the linear regime comprising weak and low-frequency (classical) fields. This alone we think is worth noting. Additionally, one outcome of our model is an independent estimate of the volume associated with a virtual pair. Furthermore, our estimates also provide an evaluation of the number of charged elementary particles. A more rigorous calculation would have to rely on quantized fields [18] and on a potentially modified response closer to the resonance frequency.

In many of the seminal nonlinear effects of the quantum vacuum, the contribution of the lightest elementary particles dominates. By contrast, the linear effects discussed here enjoy equal contributions from all elementary particles, including the ones not yet discovered, owing to the independence of the masses of the virtual particles.

Finally, the association here investigated suggests how the Maxwell equations would provide a connection between the value of the speed of light and the magnitude of the linear response of vacuum predicted by quantum physics. The situation is reminiscent of the decrease of the speed of light caused by phase shifts inside a dielectric material. We believe this to be a beautiful connection between, on the one hand, the quantum concepts of virtual particles and vacuum fluctuations and, on the other hand, the actual value of the maximum speed existent in nature baring so many fundamental consequences. The fact that the celebrated Lorentz invariant Maxwell equations would provide such a bridge just adds to the surprise.

Acknowledgements We acknowledge enlightening discussions with Katiuscia N. Cassemiro, Pierre Chavel, Joseph H. Eberly, Michael

the diamagnetic term, here denoted by $\left\langle\rho^{2}\right\rangle$ (where $\rho$ is the distance to the axis in cylindrical coordinates), originating from the actual charge distribution. Assuming a constant probability density for the electron's charge inside a solid spherical volume with radius $R$, an elementary calculation shows that $\left\langle\rho^{2}\right\rangle=2 / 5 \times R^{2}$. Using this value in (12) and the volume $V=4 \pi / 3 \times R^{3}$ in (6), we find $R=\sqrt{5 / 2} \times \hbar c /\left(\hbar \omega_{0}\right)$ by imposing $\tilde{\varepsilon}_{0} \tilde{\mu}_{0}=1 / c^{2}$. These results change $\tilde{\varepsilon}_{0}$ of (17) to the new estimate

$\tilde{\varepsilon}_{0}=3 \alpha\left(\frac{2}{5}\right)^{3 / 2} \frac{\hbar \omega_{0}}{m c^{2}} \varepsilon_{0}$.

For the total number of virtual elementary pairs contributing to the vacuum response, one finds in place of (19) the estimate

$\sum_{j}\left(\frac{q_{j}}{e}\right)^{2}=\frac{1}{3 \alpha}\left(\frac{5}{2}\right)^{3 / 2} \frac{m c^{2}}{\hbar \omega_{0}}$,

resulting in a number close to 90 pairs if $\hbar \omega_{0}=2 m c^{2}$. Finally, we note that the actual charge distribution would be probably peaked around the origin, in this manner reducing the ratio $\left\langle\rho^{2}\right\rangle / R^{2}$ and increasing as a consequence the effective volume and the number of contributing virtual pairs. 
Fleischhauer, Holger Gies, Uli Katz, Natalia V. Korolkova, Gérard Mourou, Nicolai B. Narozhny, Serge Reynaud, Wolfgang P. Schleich, Anthony E. Siegman, and John Weiner.

Open Access This article is distributed under the terms of the Creative Commons Attribution Noncommercial License which permits any noncommercial use, distribution, and reproduction in any medium, provided the original author(s) and source are credited.

\section{References}

1. D.J. Griffiths, Introduction to Electrodynamics, 3th edn. (PrenticeHall, New Jersey, 1999), p. 180

2. I.E. Tamm, Fundamentals of the Theory of Electricity (Mir, Moscow, 1979), p. 117

3. E.M. Purcell, Electricity and Magnetism. Berkeley Physics Course, vol. II (McGraw-Hill, New York, 1965), p. 332

4. M.J. Duff, L.B. Okun, G. Veneziano, J. High Energy Phys. 03, 023 (2002)
5. M. Kitano, arXiv:physics/0607056v2 [physics.gen-ph]

6. R. Pike, Propagation in spatially inhomogeneous electrical and acoustic waveguides, PT invariance and the units of vacuum impedance. (In preparation)

7. P. Milonni, The Quantum Vacuum (Academic Press, New York, 1994)

8. W. Dittrich, H. Gies, Springer Tracts Mod. Phys. 166, 1 (2000)

9. W. Heisenberg, H. Euler, Z. Phys. 98, 714 (1936)

10. F. Sauter, Z. Phys. 69, 742 (1931)

11. F. Sauter, Z. Phys. 73, 547 (1931)

12. J. Schwinger, Phys. Rev. 82, 664 (1951)

13. M. Ruf, G.R. Mocken, C. Müller, K.Z. Hatsagortsyan, C.H. Keitel, Phys. Rev. Lett. 102, 080402 (2009)

14. N.B. Narozhny, S.S. Bulanov, V.D. Mur, V.S. Popov, Zh. Eksp. Teor. Fiz. 129, 14 (2006) [JETP 102, 9 (2006)]

15. R.P. Feynman, R.B. Leyton, M. Sands, The Feynman Lectures on Physics, vol. 2 (Addison-Wesley, Reading, 1964). Chap. 34

16. L.D. Landau, A.A. Abrikosov, I.M. Khalatnikov, Dokl. Akad. Nauk 95, 1177 (1954)

17. A.D. Sakharov, Teor. Mat. Fiz. 23, 178 (1974)

18. J. Savolainen, S. Stenholm, Am. J. Phys. 40, 667 (1972) 